\title{
La aplicación del conocimiento por los trabajadores en los sistemas productivos. Un análisis desde el taylorismo, el fordismo y la producción flexible (The application of knowledge by workers in production systems. An analysis from Taylorism, Fordism and Flexible Production)
}

\section{' Erika Pedraza Sánchez \\ Esthela Gutiérrez Garza \\ Elizabeth Gálvez Santillán}

\begin{abstract}
The present article develops an analysis on the importance of the application of the knowledge on the part of the workers in the systems of production Taylorist, fordista and of flexible production. A documentary analysis is carried out and it is found that, in order of importance, the flexible production system is the one that most promotes the application of knowledge by workers, then Taylorism and finally Fordism.
\end{abstract}

Key words: knowledge and worker, taylorist production system, fordist production system, flexible production system.

JEL: D2, L2, M2.

Resumen. El presente artículo desarrolla un análisis sobre la importancia de la aplicación del conocimiento por parte de los trabajadores en los sistemas de producción taylorista, fordista y de producción flexible. Se realiza un análisis documental y se encuentra que, en orden de importancia, el sistema de producción flexible es el que más promueve la aplicación del conocimiento por parte de los trabajadores, después el taylorismo y por último el fordismo.

Palabras clave: conocimiento y trabajador, sistema de producción taylorista, sistema de producción fordista, sistema de producción flexible. 


\section{Introducción}

De acuerdo a David y Foray (2002) históricamente el conocimiento ha sido uno de los principales elementos en el crecimiento económico de un país o región, pues es a través de este que se puede generar la capacidad de inventar, de innovar, para materializarse luego en nuevos productos, procesos u organizaciones lo que a su vez incentiva el desarrollo económico.

Sin embargo, al hablar de manera particular de los procesos productivos, la importancia que se le ha dado al aspecto del conocimiento de los trabajadores en el proceso de producción no siempre ha sido la misma.

Conocimiento es "una capacidad cognoscitiva; esto es, que comprende las capacidades de ampliar, extrapolar e inferir nueva información para realizar actividades intelectuales o manuales" (David y Foray, 2002:7).

Además un proceso productivo tiene por esencia la creación de riqueza 0 de valor en términos de alcanzar altos niveles de productividad y competitividad, por lo que se van estructurando una serie de técnicas que dan lugar a un determinado sistema de producción con características en base al nivel de desarrollo tecnológico, división del trabajo y procesos de distribución y organización (Gutiérrez, 2009).

El presente artículo hace un análisis de la importancia del conocimiento que tienen los trabajadores para el desarrollo de sus actividades en el proceso productivo, con el fin de conocer cuál de estos sistemas promueve más la aplicación de los conocimientos y la creatividad de los trabajadores en las actividades que desarrollan en cada sistema productivo. El análisis se realiza en los tres sistemas de producción de empresas más utilizados del siglo XX a la fecha: el taylorismo, el fordismo y el de producción flexible.

Estos sistemas de producción han surgido de manera paulatina, siendo el más antiguo el taylorismo y el más nuevo la producción flexible, no obstante, ninguno ha desaparecido e incluso se han generado modelos híbridos como los sistemas fordtayloristas, organizados sobre la fragmentación del trabajo con puestos individuales fijos y repetitivos, con las cadenas de montaje típicos del fordismo, junto con los sistemas de producción flexible que son integrados por máquinas de control numérico incorporadas en el proceso de trabajo para flexibilizar la línea de producción (Gutiérrez 2009).

En general, la bibliografía que existe en torno a los sistemas de producción, abordan la operación de los modelos productivos, por ejemplo, Córdoba 
(2006), Ramírez (1989), Rivero (2002) y Zuccarino (2012); este trabajo pretende hacer una aportación desde la aplicación del conocimiento de los trabajadores en el sistema productivo.

Se trabaja bajo la hipótesis de que las posibilidades de aplicar nuevo conocimiento por parte de los trabajadores a las actividades que realizan es prácticamente nula en los primeros dos modelos de producción y que, contrario a esto, en el tercero de los sistemas, este aspecto es un elemento esencial para elevar la productividad.

Este trabajo es una investigación documental y se organiza de la siguiente manera: en el primer apartado se describe de manera general el sistema de producción taylorista, para conocer cómo se organizan los factores productivos, de manera particular el trabajador y la forma como aplica el conocimiento en sus actividades productivas; en el segundo apartado se desarrolla el modelo de producción fordista y en el tercer apartado el sistema de producción flexible; posteriormente, al final, se detallan los resultados y las conclusiones comparando la aplicación del conocimiento por los trabajadores en los tres sistemas productivos.

\section{Sistema de producción taylorista}

El método de administración científica, conocido como sistema de producción taylorista, surge a finales del siglo XIX y fue creado por el ingeniero estadounidense Frederick Taylor, quien señalaba que un sistema de producción eficiente y productiva debe estar basado en los principios de la administración cientíica, esto es, en "leyes, reglas y principios definidos" y no en métodos empíricos.

Para Taylor este razonamiento sobre el sistema de producción asegura que se cumple el propósito "del máximo de prosperidad al empleador, unido al máximo de prosperidad para cada empleado", pues además de incrementar las ganancias de las empresas se expanden a su nivel máximo sus capacidades y funciones; mientras que por el otro lado, el trabajador, aparte de poder ganar mejores salarios, desarrolla y maximiza su eficiencia para efectuar un trabajo apropiado a su capacidad natural.

El modelo de la administración científica se basa en cuatro principios fundamentales: el primero, desarrollar cada elemento del trabajo a través de la ciencia y no en una regla empírica; el segundo, instruir al obrero diariamente por sus superiores para efectuar su trabajo; el tercero, la cooperación entre la administración y los obreros; y el cuarto, distribución equitativa de la responsabilidades en 
tareas entre los mismos (Taylor, 1981:29). En otras palabras, el modelo establece como punto de partida que "la administración tiene la obligación de recopilar los métodos de trabajo tradicionales empleados por los obreros, clasificarlos, tabularlos y deducir de ellos las reglas, leyes y fórmulas que guiarán en lo sucesivo a los obreros en su tarea diaria", luego "el trabajo de cada obrero es preparado enteramente por la administración, con instrucciones escritas detallando la tarea a realizar y qué procedimientos habrá de realizar", posteriormente, es esencial "la cooperación personal estrecha con los obreros para que todo el trabajo sea realizado con los principios científicos que se aplican" y, por último, "la administración asume todo trabajo que exceda la capacidad de los obreros, mientras que en el pasado casi todo el trabajo y la mayor responsabilidad era confiado a este", de esta manera, "existe una división mucho más equitativa de la responsabilidad entre la dirección y los obreros, no obstante es responsabilidad completa de la administración la planeación, control y diseño de las actividades productivas y no del trabajador individual" (Taylor, 1981:29).

Bajo este modelo se organizaba el taller (lugar de trabajo) y el trabajo sobre una nueva base científica denominada cronómetro, definido y vigilado por la empresa, por medio de tablas de tiempos y movimientos en cada etapa del proceso productivo. El cronómetro asegura "la integración progresiva de los trabajadores no especializados en los puestos de los profesionales de oficio" (Coriat, 2005: 36-37). Por lo que el modelo cumple su función al disminuir los tiempos innecesarios de los trabajadores, y se "apodera del saber hacer del obrero: conocimientos y habilidades transmitidos desde tiempos ancestrales, de generación en generación en sus estudios de tiempos y movimientos. (Ramírez, 1989:80)

En ese sentido, el propósito del taylorismo es aumentar la productividad con base en la máxima división de funciones, la especialización del trabajador y el control estricto de tiempo para la realización de cada tarea, además de ligar la remuneración del trabajador a su rendimiento, es decir, a la productividad. De esta manera el sistema resta importancia al beneficio que pueda obtener del conocimiento o creatividad de los obreros al proceso productivo, la aplicación del conocimiento es concebido y desarrollado únicamente por los jefes de administración o ingenieros para el diseño y creación de tareas o cargos conforme mejor funcione, limitando al obrero solo a realizar la función indicada por la organización en su momento.

La técnica empleada es bajo la supervisión de los jefes de administración en la ejecución de las tareas diarias y del sistema de incentivos salariales, 
contrario a lo que ocurría con el sistema previo de administración donde al obrero se le dejaba el control total de los tiempos de producción para efectuar su oficio 0 tarea, lo que no aseguraba que se logrará la mayor productividad.

\section{Sistema de producción fordista}

El sistema de producción fordista surge en Estados Unidos en las dos primeras décadas del siglo XX, aunque es hasta finales de la década de 1930 cuando se convierte en un modelo de producción industrial y, a partir de ahí, se generaliza a todos los países. Su denominación proviene del creador de la línea de ensamble, Henry Ford.

Aunque la base de este modelo sigue siendo la administración científica de Taylor, el incremento de la productividad se logra gracias a dos aspectos: la incorporación de la cadena de montaje al proceso productivo y a la división de tareas entre los obreros en su máxima expresión, lo que da lugar a la producción en serie (Safón, 1997). Lo que muestra que en este modelo el conocimiento de los trabajadores continúa siendo no relevante para la empresa.

El fordismo es "la producción en serie en el modelo de cadena de montaje, al utilizar maquinaria con fines especiales y, principalmente, trabajadores no cualificados en una división del trabajo basada en una fragmentación de tareas cada vez mayor. La era fordista se caracteriza por la dominación de los mercados de masas y por bienes estandarizados que se mantienen durante largo tiempo. El fordismo surge de la eficiencia tecnológica de la producción planificada, que se basa en la separación entre concepción y ejecución, y de la eficiencia económica de fábricas de gran escala. El fordismo viene dominado por la lógica económica, la ventaja comparativa y del funcionamiento del mercado" (Hirst y Zeitlin 1991; citado en Safón 1997).

Otra de las particularidades del modelo de producción fordista es el desarrollo de innovaciones continuas en el interior de la fábrica, como los trasformadores de cintas y de cadena, las grúas de puente y máquinas especializadas cada una vinculada a su línea de producción, lo que induce a no ser un modelo estático sino en constante movimiento. Al ser regulado de forma electrónica, el transportador fija la velocidad deseada al proceso, estableciendo el tiempo y movimientos para cada una de las operaciones. De esta manera, el modelo fordista disminuye el sobrante de las materias primas y origina la producción en masa. (Ramírez, 1989) 
Enseguida se resumen las características más relevantes de este modelo de automatización rígida, de acuerdo a Ramírez (1989:80):

- La rigidez de los equipos utilizados en el sistema de producción (cadena de montaje).

- La poca o nula diversificación de la producción, solo se contempla a largo plazo.

- La aplicación de las economías de escala en el proceso, por lo que se renuncia a la flexibilidad del producto.

- La rápida obsolescencia del equipo especializado y del producto semielaborado (almacenados y en proceso).

Piore y Sabel (1990:36) sostienen que con la introducción de la cadena de montaje se invierten los papeles del artesano y el instrumento, esto es, "el trabajador se convirtió en un apéndice de la máquina, cuyo propósito, lejos de traducir las cualificaciones humanas en actos, resulta superflua la participación del hombre en la producción de los bienes", situación que refleja la supremacía de la producción bajo cualquier situación ante el trabajador.

Aguirre (2007:29) señala que en esta nueva concepción tecnológica se articulan todas las fases del proceso del producto final, mecanizada en su totalidad. Lo que hace que las tareas individuales de los obreros sean simples movimientos de rápida y fácil ejecución y ante "un mercado de gran escala, de producción en serie y estandarización, permite dividir o pulverizar el trabajo en tareas cada vez más subdivididas", de esta manera "se eliminan los tiempos improductivos, regulando la forma específica de su tarea individual" ya no por medio de capataces o primas salariales, cómo funciona en el sistema taylorista, sino a través de las propias máquinas. (Aguirre, 2007; Safón, 1997).

Coriat (2005:107) menciona que a raíz de esta modernización de los aparatos productivos, exige cada vez más demandar una fuerza de trabajo considerada no cualificada, dedicada a tareas simples de ejecución o capacitada en la empresa en pocas semanas. Consecuencia de las propias modificaciones en la división del trabajo y del maquinismo que llevan a un doble proceso: "el de separación entre el trabajo de concepción y el trabajo de ejecución; y el de parcelación y repetitividad en los propios trabajos de concepción y ejecución".

Así, en el modelo fordista, la productividad se origina a través de la producción masiva de unidades de producto con base en la tecnología de ensamblaje o producción en serie, capaz de reducir costos y con excedentes de lo producido mayores a la capacidad de consumo (Gutiérrez, 1985 y 2009). 
En conclusión, dada la configuración que contempla este sistema es evidente que no se aprovecha el conocimiento o el talento del trabajador para la mejora o iniciativa en alguna fase del proceso productivo, solamente el conocimiento que es aprendido o adquirido en pocas semanas de capacitación dentro de la organización para desempeñar la actividad determinada es suficiente, lo que conduce a un trabajador mecanizado en funciones y alejado de toda creatividad.

\section{Sistema de producción flexible: el toyotismo}

Un sistema de producción flexible tiene su fundamento en "la capacidad de programación del equipo productivo, en las técnicas que permiten la interconexión entre equipos que cumplan funciones diversas y en la posibilidad de actuar en tiempo real". Estos sistemas surgen en la década de 1970 para dar respuesta a la rigidez de los sistemas tayloristas-fordista y en la búsqueda de incrementar la productividad. (Ramírez, 1989:75)

Ramírez (1989:75) señala que este sistema "es capaz de producir una diversidad de bienes con variedad en la composición del producto que puede asimilar rápidamente las innovaciones y que requiere de la coordinación continua y profunda de equipos, procesos y recursos humanos". Tales sistemas se pueden aplicar "sin intervención humana en verificación de averías, diagnostico de fallos y corrección de errores" (Bañegil, 1991: 68-69).

Algunas modalidades de producción flexible son el uso de los robots industriales, los almacenes automatizados, el just in time, las máquinas herramientas de control numérico, círculos de control de calidad y las computadoras en los procesos de diseño y manufactura (Ramírez, 1989:81). El surgimiento de estos sistemas de producción principia con los avances tecnológicos en la microelectrónica, que permitió dar ese empuje como un instrumento de producción y que más tarde converge en la existencia de sistemas de producción con modalidad flexible alternando con formas de trabajo y de producción distintas.

En ese sentido, uno de los avances tecnológicos más innovadores en la microelectrónica, es el microprocesador u ordenador en un chip realizado a principios de los años de 1970, por un ingeniero de la región de California, específicamente en Silicon Valley. Fue en este momento que la microelectrónica se expande por completo a la población en general, es decir, se inicia la comercialización de esta tecnología capaz de procesar información y de instalarse en todas partes. Castells (1999: 68) 
Coriat (2007:82) añade que es precisamente el uso científico y técnico el que logra dar soporte a la creación de equipos flexibles sustentados en la microelectrónica, con lo cual se comienza a trazar una alternativa como fuente de productividad, destacando la presencia de "los sistemas de máquinas de control numérico, los equipos computarizados con diseño y fabricación integrados, y los robots".

Radovan Richta (1970), plantea que la celeridad en el desarrollo de la ciencia y la técnica provoca una transformación universal de todas las fuerzas productivas, al grado de que es capaz de modificar, en esencia, a todo el proceso productivo, no únicamente al avance continuo de un factor de producción, sino también al capital humano y, con ello, otorga al hombre una nueva postura, capaz de permear en toda la sociedad, en todos sus aspectos económicos, sociales y culturales. Esto es, la aplicación de la ciencia y la técnica en los procesos de producción es "el saber acumulado de la sociedad" el fundamento esencial y "factor decisivo de la evolución y crecimiento de las fuerzas productivas". (Ritcha, 1970:14).

De esa manera, Coriat (2005:33) sostiene que "la informática, la electrónica, las telecomunicaciones y la robótica se entrelazan en una red nueva fuertemente interactiva de fuerzas productivas de grandes potencialidades concernientes a los progresos en productividad de los que son portadoras 0 en la redefinición del contenido y calidad de uso de los productos".

Leborgne y Lipietz (1992:19) concilian, al señalar que estas "nuevas tecnologías son capaces de ofrecer una respuesta a las necesidades de flexibilidad de la demanda de bienes", no obstante también es necesaria la introducción de una fuerza de trabajo especializada para el manejo de las mismas.

Estos cambios de concepción tecnológica son los que suscitan transformaciones radicales en el proceso de trabajo. Coriat (2011:150) señala que estos métodos "instauran un proceso de intelectualización y de blanqueamiento de los cuellos azules". Es así como nace y se consolida un modelo general de trabajo diferente, "un modelo de competencia, plenamente asumido y alimentado por la valorización en las habilidades y calificaciones; fabricantes, tecnólogos y administradores como nuevas figuras clave de la empresa" (Coriat, 2005: 203).

Gutiérrez (2009:63) indica que "el uso de tecnologías flexibles requiere de una planta laboral mejor calificada, y de un mayor involucramiento para la aplicación de programas de calidad que las tecnologías informatizadas necesitan", además que en el uso de los equipos flexibles, como el control numérico y robots 
"es necesario el involucramiento del trabajador, el trabajo en equipo y la mejora continua".

Ahora bien, para efectos de esta investigación, se describe brevemente a uno de los sistemas de producción flexible más conocido y proveniente de Japón, el modelo Just in time, es decir, Justo a tiempo o de producción en flujo continuo. Este sistema de producción surge en la década de los 70, se denomina toyotismo u onhismo por el nombre de su creador, Taichí Ohno, vicepresidente de la empresa japonesa Toyota Motor Company. El proceso Just in time se define como "la producción consistente en producir la cantidad de producto exigida por el mercado al nivel de calidad deseado, en el momento preciso, -sin generar excedentes en la empresa" (Albizu, 1996: 102). En otras palabras, el sistema justo a tiempo significa que "en un proceso continuo, las piezas adecuadas necesarias para el montaje deben incorporarse a la cadena de montaje justo en el momento en el que se necesitan y donde el stock generado es nulo" (Albizu, 1996:103)

La técnica del kanban es el método operativo del Justo a tiempo que consiste en "una tarjeta que introducida en una funda de plástico transparente contiene información sobre traslados, entregas o fabricación principalmente. El kanban se utiliza para indicar a la máquina/operación anterior que necesita más trabajo 0 bien proporcionar a la línea de producción la información precisa para comenzar a producir" (Albizu, 1996:103). Así las piezas se producen en la cantidad que señalan los kanban; por lo que el número de kanban en existencia o en funcionamiento determinará el nivel máximo de producción a realizar. Según Ramírez (1989), Taichi Ohno, después de varios esfuerzos en investigar las funciones innecesarias en las operaciones de producción, logró sintetizar las ideas desarrolladas por los trabajadores de todos los niveles, creando la técnica de KanBan como un medio de la producción justo a tiempo.

La base del sistema de producción Toyota reside en ciertos principios básicos integrados al proceso productivo que aspiran en alcanzar el máximo rendimiento de las unidades productivas. Albizu (1996) señala que hay autores que los denominan postulados, formando la teoría de los cincos ceros (cero defectos, cero detención de la producción, cero inventario, cero papel y cero demora). El autor los resume como sigue:

- Reducción de inventarios.

- Reducción de los defectos.

- Reducción de tiempos de preparación de máquinas y averías.

- Reducción de tiempos de transporte de piezas. 
Ohno (1991, citado en Albizu 1996) señala que "la mayor de las improductividades es el exceso de stock", debido a las implicaciones en costos en "almacenamientos, espacio, recursos económicos inmovilizados, trabajadores encargados de su transporte y mantenimiento, elementos de transporte, oxidaciones y obsolescencias". (Albizu, 1996:107).

Para el logro de una producción sin defectos se introduce el concepto de calidad total. Según Albizu (1996:108), este elemento se incorpora desde la etapa de diseño de producto y continua en el proceso de fabricación; "se utilizan máquinas que producen piezas de calidad uniforme y se lleva a cabo una comprobación continua en la línea de producción mediante sistemas automáticos y por el propio personal de la fábrica". Para entendimiento del concepto de calidad, el autor lo define como "la eliminación de despilfarros o todo aquello que no añada valor". Así, es necesaria la visión y el compromiso de todo el equipo de trabajo en inspeccionar el proceso productivo en todas las etapas y tomando las medidas correctivas necesarias (Albizu, 1996); para lograrlo, el conocimiento y creatividad de los trabajadores se vuelve importante.

En el sistema de producción justo a tiempo, la reducción de tiempos de preparación de máquinas y averías debe ser mínima y requiere "un importante cambio de actitud de toda la gente de la fábrica" (Albizu 1996:110). El concepto de tiempo de preparación o tiempo de cambio se entiende como "el lapso que transcurre desde la salida de la última unidad producida de un tipo y la primera unidad producida de otro tipo o modelo". Onho (citado en Albizu, 1996:110) señala que "el valor de una máquina no debe determinarse por sus años de servicio o edad. Se determina por el potencial de generación de beneficios que todavía posee", por lo que es necesario capacitar a los trabajadores de la fábrica en programas permanentes de mantenimiento preventivo (limpiezas, revisiones) para el cuidado de las máquinas. (Albizu, 1996).

Por último, la etapa de la reducción de tiempo de transporte de piezas se logra a través del sistema kanban y a la exigencia a los proveedores de suministrar el flujo de materiales en la fábrica acorde a los tiempos acordados. Por lo que es fundamental la posición física de los puestos de trabajo que facilite el trayecto de los materiales y de las piezas en el interior de la fábrica, que resulte mínimo el transporte entre las operaciones. (Albizu, 1996)

Ramírez (1989:98) señala que el fin de este modelo implica producir productos en la cantidad y el tiempo necesario, dando como resultado la disminución en excedentes de inventarios y trabajo, cumpliendo así "los propósitos de 
incrementar la productividad y reducir costos". Para Albizu (1996:108) el sistema justo a tiempo "pretende la reducción de los inventarios como la búsqueda y solución de los problemas de producción".

Coriat (2007:94) menciona que el kanban como subsistema del justo a tiempo representa una auténtica "innovación organizacional, que traza su diferencia con relación a los grandes principios taylorianos de la separación funcional del trabajo" (Coriat, 2007:94).

Gutiérrez (2009:65) señala que este sistema productivo dicta "el principio del involucramiento del trabajador y del ejercicio intelectual con el sistema kanban". Esto es, un sistema de producción que, acorde a sus características de origen como es la aplicación de nuevas tecnologías, equipos computarizados en diseño y fabricación y uso de robots industriales, contemple la necesidad de un "tipo de trabajador multifuncional con mayor intervención en todas las fases de producción, capaz de alcanzar la transcendencia de la producción flexible y de calidad". Para el autor, las características de los sistemas taylorista y fordista "priorizan más al capital o equipos de tecnología rígida e inflexible, limitando al trabajador a un proceso de mecanización e individualización”. (Gutiérrez, 1985, 1996, 2009).

\section{Metodología}

Este trabajo se basa en una revisión documental, la cual entra en la categoría de análisis cualitativo. Se lleva a cabo una comparación entre los diferentes sistemas de producción para conocer la importancia que se le da al conocimiento de los trabajadores en los procesos de producción.

De esta manera, la presente investigación es un estudio de tipo exploratorio y descriptivo; pues de acuerdo a Hernández, Fernández y Baptista (2014) es un diseño transeccional descriptivo, cuyo objetivo es conocer la incidencia de una variable en una población, ya sea un grupo de personas, objetos, situaciones, contextos, fenómenos, comunidades etc., y así poder proporcionar su descripción.

En este caso es en determinar la importancia del conocimiento de los trabajadores en los procesos de producción taylorista, fordista y de producción flexible; por lo que este estudio es descriptivo (o entra en una categoría de descriptivo metodológicamente hablando). 


\section{Resultados}

Una vez concluida la revisión documental, a continuación se presentan los resultados obtenidos acerca de las características del tipo de perfil del trabajador y su aplicación del conocimiento en el desarrollo de sus actividades en cada sistema de producción (ver Tabla 1).

Tabla 1. El perfil del trabajador y su aplicación del conocimiento en los sistemas de producción taylorista, fordista y de producción flexible

\begin{tabular}{|c|c|c|}
\hline \multicolumn{3}{|c|}{ Sistema de producción } \\
\hline Taylorismo & Fordismo & Producción flexible \\
\hline $\begin{array}{l}\text { Solo se valora el trabajo } \\
\text { manual del trabajador y se } \\
\text { excluye su capacidad de obra } \\
\text { basada en su conocimiento } \\
\text { y creatividad. }\end{array}$ & $\begin{array}{l}\text { Sólo importa el trabajo } \\
\text { manual y se excluye por } \\
\text { completo la capacidad } \\
\text { intelectual del trabajador. }\end{array}$ & $\begin{array}{l}\text { El trabajo manual } \\
\text { y el intelectual están } \\
\text { fuertemente asociados } \\
\text { y valorados. }\end{array}$ \\
\hline $\begin{array}{l}\text { Trabajo fragmentado } \\
\text { contratado para realizar } \\
\text { actividades repetitivas, fijas } \\
\text { cuyo desempeño individual } \\
\text { es instruido y controlado por } \\
\text { la empresa y supervisores. }\end{array}$ & $\begin{array}{l}\text { Trabajo fragmentado en } \\
\text { mayor intensidad. Trabajo } \\
\text { repetitivo, fijo y desempeño } \\
\text { individual ahora regulado en } \\
\text { forma mecánica, es decir, } \\
\text { por la cadena de montaje, } \\
\text { principalmente. }\end{array}$ & $\begin{array}{l}\text { Trabajo integrado. } \\
\text { Contratado para efectuar } \\
\text { trabajos complejos, } \\
\text { diversificados y en equipo. }\end{array}$ \\
\hline $\begin{array}{l}\text { Desprofesionalización } \\
\text { del trabajo. Trabajo simple, } \\
\text { especializado o fragmentado. }\end{array}$ & $\begin{array}{l}\text { Desprofesionalización } \\
\text { del trabajo. El trabajo es } \\
\text { cada vez menos cualificado. }\end{array}$ & $\begin{array}{l}\text { Profesionalización } \\
\text { del trabajo. Se requiere } \\
\text { de trabajo abstracto, } \\
\text { calificado y multifuncional. }\end{array}$ \\
\hline $\begin{array}{l}\text { Trabajador separado } \\
\text { y aislado. La empresa } \\
\text { controla el saber hacer } \\
\text { y todo el proceso productivo. }\end{array}$ & $\begin{array}{l}\text { Trabajador separado } \\
\text { y aislado. La empresa } \\
\text { controla el saber hacer y } \\
\text { todo el proceso productivo }\end{array}$ & $\begin{array}{l}\text { Trabajador involucrado. } \\
\text { La condición del trabajador } \\
\text { en la planta es activa, } \\
\text { participativa y creativa en } \\
\text { el proceso productivo. }\end{array}$ \\
\hline $\begin{array}{l}\text { El sistema productivo resta } \\
\text { importancia al conocimiento } \\
\text { o creatividad de los trabaja- } \\
\text { dores que pudieran aportar } \\
\text { al proceso productivo. }\end{array}$ & $\begin{array}{l}\text { El sistema no aprovecha } \\
\text { el conocimiento o el talento } \\
\text { del trabajador para la mejora } \\
\text { o iniciativa sobre el proceso } \\
\text { de productivo. }\end{array}$ & $\begin{array}{l}\text { Para este sistema es } \\
\text { fundamental la aplicación del } \\
\text { conocimiento y creatividad de } \\
\text { los trabajadores como un } \\
\text { elemento esencial para } \\
\text { elevar la productividad. }\end{array}$ \\
\hline
\end{tabular}

Fuente: Elaboración propia 
De acuerdo a la Tabla 1, en el caso del sistema de producción taylorista el conocimiento se vuelve clave para el área administrativa de la empresa; mientras que el trabajador no instruido pasa a ser el elemento ideal para la realización de las actividades operativas del proceso de producción, ya que para la empresa es mejor enseñar a alguien desde el inicio que a alguien que ya tiene un conocimiento de cómo hacer las cosas debido a que quizás no quiera cambiar su forma de trabajar, es decir, bajo este modelo, el conocimiento del trabajador aún es importante, aunque de manera parcial, pues dependerá de la actividad que realizará el conocimiento que le impartirá la empresa. Bajo este modelo, el conocimiento de cómo hacer las cosas es únicamente cuestión de la empresa.

En el sistema fordista desaparece cualquier posibilidad de aplicación de conocimiento al proceso productivo por parte del trabajador; el cual queda totalmente sometido a lo que dicta la cadena de montaje (Piore y Sabel 1990). Con la acentuación de la división de tareas de los trabajadores y el dominio del proceso productivo por las máquinas, este tipo de modelo exigió cada vez más mano de obra no cualificada, pero además con la simplificación de tareas para el desarrollo de su trabajo no requería en general una capacitación compleja. En este modelo, el conocimiento se reserva para las esferas más altas de la empresa.

Para el sistema de producción flexible se encuentra que el conocimiento de los trabajadores es uno de los elementos claves, junto con otros aspectos como la tecnología, etcétera; para el logro de los resultados deseados, como el incremento de productividad, incluso el trabajador adquiere una mayor apreciación de todo el proceso productivo.

\section{Conclusiones}

En el recorrido que se ha realizado en torno a los procesos productivos taylorista, fordista y de producción flexible se puede observar cómo la importancia del conocimiento en el trabajador, para el desarrollo de sus actividades laborales, cambia de un modelo productivo a otro.

Con el análisis de esta revisión documental se está en condiciones de poder evaluar la hipótesis planteada al inicio del artículo, donde las posibilidades de aplicar el conocimiento por parte de los trabajadores en sus actividades productivas es prácticamente nula en los primeros dos sistemas de producción y mayor en el sistema de producción flexible. De esta manera, con base en los resultados obtenidos, se puede indicar que. por orden de importancia, la producción flexible 
es el sistema que más promueve la aplicación del conocimiento por parte de los trabajadores en los procesos de producción, seguida del sistema taylorista y por último el sistema fordista.

Los sistemas de producción taylorista y fordista están soportados por la misma base científica propuesta por Taylor, en ambos sistemas emplean el mismo tipo de perfil del trabajador o bien, con las mismas características al demandar una fuerza de trabajo con escasa cualificación para el desarrollo de las actividades en la empresa, con la diferencia que en el segundo sistema se incorpora la cadena de montaje.

En el sistema de producción flexible al estar sustentado por máquinas y equipos computarizados que facilitan este tipo de producción, la aplicación del conocimiento o la creatividad del trabajador se vuelve clave para el buen funcionamiento del proceso productivo y para el incremento de la productividad.

\section{Referencias}

Aguirre, Carlos. (2007). Taylorismo y Fordismo: formas de hiperracionalización del trabajo. Revista Mundo Siglo XXI, 11, 23-43.

Albizu, Eneka (1996). La producción flexible: Just in time vs. Organización Científica del Trabajo. Revista de Dirección y Administración de Empresas, 4.

Bañegil Palacios, Tomás M (1991) La flexibilidad de la producción y el sistema just in time en España. Tesis Doctoral. Universidad Autónoma de Madrid. Madrid, 1991.

Castells, Manuel (1999). La era de la información. Economía, sociedad y cultura. Volumen I. México. D.F. Editorial Siglo XXI.

Córdova, Ernesto (2006). Manufactura y automatización. Revista Scielo, 26(3).

Coriat, Benjamín (2005). El taller y el cronómetro. Ensayo sobre el taylorismo, el fordismo y la producción en masa. Siglo XX Editores. México, D.F.

Coriat, Benjamín (2007). El taller y el robot. Ensayos sobre el fordismo y la producción en masa en la era de la electrónica. Siglo XXI Editores. México,D.F.

Coriat, Benjamín (2011). Pensar al revés. Trabajo y organización en la empresa japonesa. México, D.F. Siglo XXI Editores.

David, Paul y Foray, Dominique (2002) Una introducción a la economía y a la sociedad del saber. Revista Internacional de las ciencias sociales, 17.

Gutiérrez Garza, Esthela (1985). Restructuración productiva y clase obrera. Testimonios de la crisis. Siglo XXI Editores, 9-60.

Gutiérrez, Esthela. (1996). Los polos dinámicos de la industrialización. La industria moderna y la industria maquiladora. Nuevo León: 1988-1995. Revista Estudios Demográficos y Urbanos, 11(2), 291-317. 
Gutiérrez, Esthela (2005) Restructuración productiva en la manufactura Nuevo León, década de los noventa. Región Industrial de Monterrey. Revista Humanitas. Anuario del Centro de Estudios Humanísticos. Edición 32. UANL.

Gutiérrez Garza, Esthela (2009). Escenarios de sustentabilidad industrial. Nuevo León 19882004. México, IINSO-UANL. Editorial Plaza y Valdés.

Hernández, Roberto; Fernández, Carlos y Baptista, Pilar (2014). Metodología de la investigación. Sexta Edición. México. Editorial Mc-Graw Hill.

Leborgne, Danielle y Lipietz, Alain. (1992). Ideas falsas y cuestiones abiertas sobre el posfordismo. Revista Trabajo, Sociedad, Tecnología y Cultura, 8, 17-31.

Piore J. Michael y Sabel F. Charles (1990). La segunda ruptura industrial. Madrid, Alianza.

Ramírez, Hilda A (1989). Sistema de producción flexible: El caso de la industria automovilista mexicana. Trabajo realizado para el Congreso de Investigación de Ciencias Sociales de la Universidad Autónoma de México, Xochimilco. Diciembre 1989.

Richta, Radovan (1970). La civilización en la encrucijada. Editorial Siglo XXI, México.

Rivero, Arturo (2002) Óscar F. Contreras (2000). Empresas globales, actores locales: producción flexible y aprendizaje industrial en las maquiladoras. Revista región y sociedad 14 (23), en http://www.scielo.org.mx/scielo.

Safon, Vicente (1997). ¿Del Fordismo al Postfordismo? El advenimiento de los nuevos modelos de organización industrial. Universidad de Valencias. I Congreso de Ciencia Regional de Andalucía: Andalucía en el umbral del siglo XXI.

Taylor, Frederick (1981). Principios de la administración científica. Argentina, Editorial El Ateneo.

Zuccarino, Maximiliano (2012) Modelos estadounidense-fordista y japonés-toyotista: ¿Dos formas de organización productiva contrapuestas? Revista región y sociedad, VII, No. 21. 